\title{
Beta-Propeller Protein-Associated Neurodegeneration Syndrome
}

\author{
Shahin Asadi*, Mahsa Jamali, Gholnesa Valizadeh and Neda Ghafouri \\ Director of the Division of Medical Genetics and Molecular Research, Iran
}

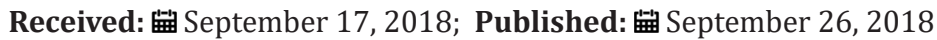

*Corresponding author: Shahin Asadi, Molecular Genetics, Director of the Division of Medical Genetics and Molecular Research, Tabriz, Iran

\section{Generalizations of BPAN Syndrome}

BPAN syndrome is a genetic disorder that progressively affects the nervous system and progressively worsens. People affected by this syndrome produce iron in the brain, which can be seen with medical imaging. For this reason, BPAN syndrome is classified with an impairment called Brain Neurodegeneration (NBIA).

\section{Signs and Symptoms of BPAN Syndrome}

Many people with BPAN syndrome have frequent seizures (epilepsy) that begin at birth or are children. There are several types of seizures in this disorder, even in a person with this syndrome. Often the first type of seizure affects the fever that is caused by the high fever. Individuals affected by BPAN syndrome may also experience tonic-clonic seizures (also known as major harmful seizures). This type of seizure affects the entire body, causing muscle stiffness and loss of consciousness. Other types of seizures that may occur in this disorder include short-term consciousness that may cause the appearance of narrow-eyed eyes or small harmful seizures, atonic antecedent seizures, involuntary muscle stretch (myoclonic seizures) or epileptic spasms. Some people with BPAN syndrome develop seizure patterns similar to those seen in epileptic syndromes such as West End syndrome or Lennox-Gastaut syndrome. Children with BPAN syndrome also have mental disabilities. Delay in the development of some skills includes vocabulary learning and speech production (language expressing) and coordination of movements (ataxia) in the syndrome. Ataxia can affect walking ability and everyday motor skills such as using living utensils. Affected people can have behavioral changes that are often compared to the characteristics of the disorder called Rett syndrome. These features include repeated hand movements or hand stereotypic movements, squeezing teeth together (brooksim), sleep disorders and communication problems, and social interaction characteristic of autism spectrum disorders.

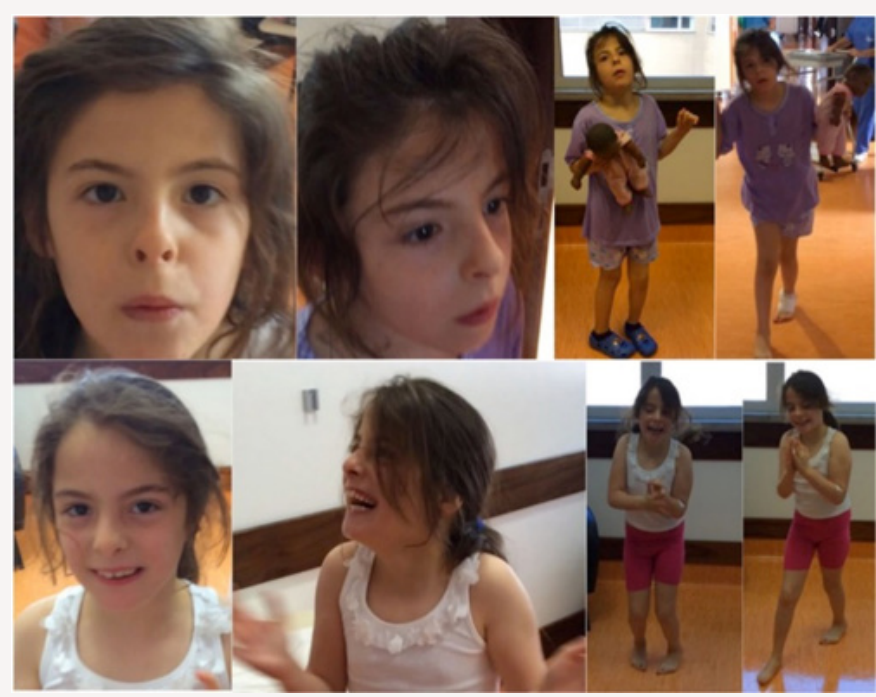

Figure 1: Images of a child with BPAN syndrome with related disorders. 
In the late adolescence or before adulthood, people with BPAN syndrome may begin to lose consciousness (cognitive decline), which can lead to intense loss of thinking and the ability to reason (intellectual decline). Difficulty of movement including dystonia and Parkinsonism also worsen. Dystrobin is a disease characterized by involuntary muscle contraction Figure 1. In BPAN syndrome, dystrophy often starts in the arms. Parkinson's can include involuntary motion (bradycinsis), muscle stiffness, tremor, inability to keep the body properly and balanced (local instability) and walking along the way, which can cause repeated falls or Repeat falling. The life span of people with BPAN syndrome varies. With proper management of their symptoms and symptoms, people with this disease can live up to middle age. Death may be due to mental retardation or motor problems, such as damage from falling or difficulty swallowing (dysphagia). Dysphagia can lead to bacterial pulmonary infection called aspiration pneumonia.

\section{Etiology of BPAN Syndrome}
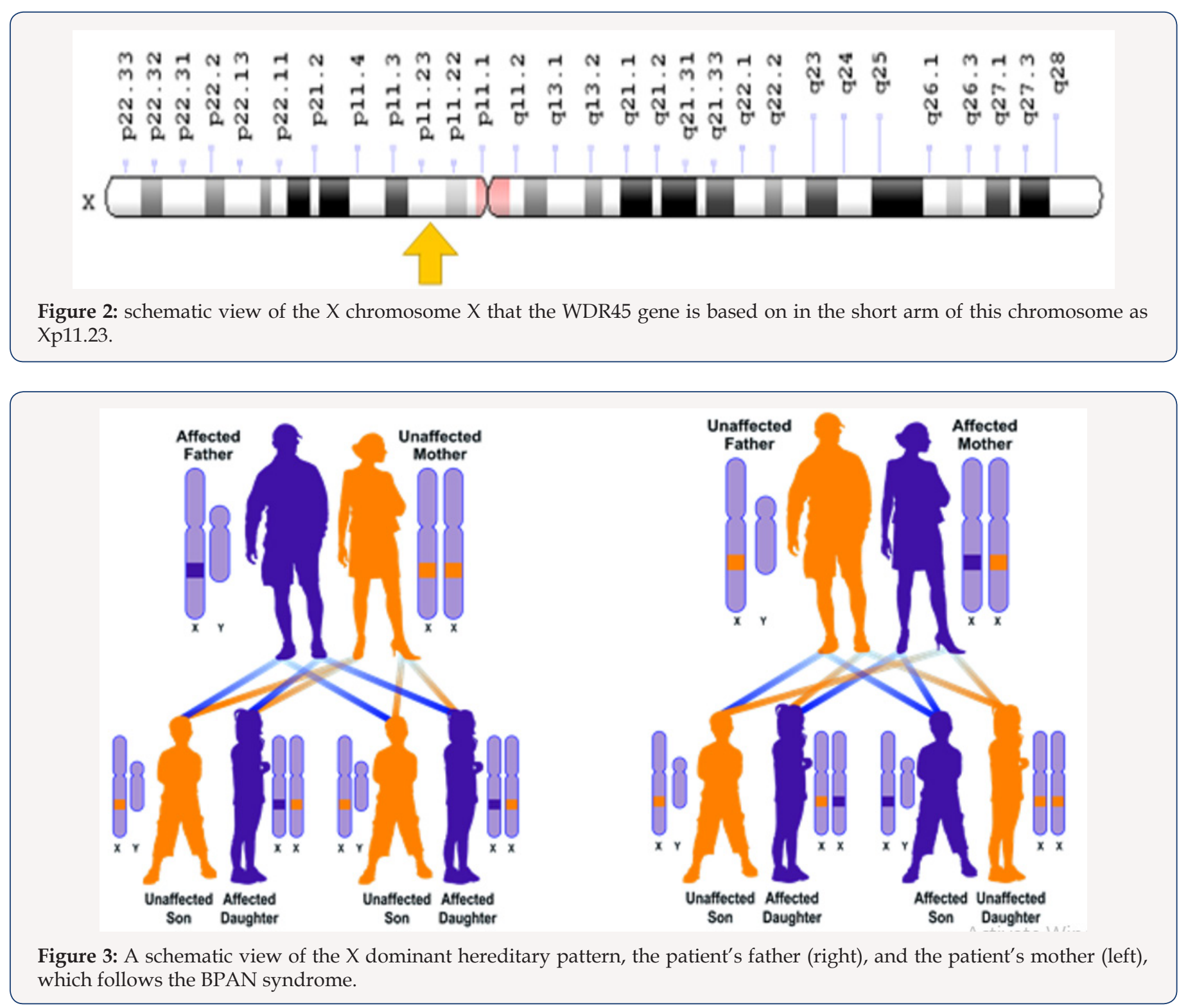

BPAN syndrome is caused by the mutation of the WDR 45 gene, which is based on the X-shaped X-chromosome $\mathrm{X}$ arm as Xp11.23. This gene provides instructions for the synthesis of the WIPI4 protein. The WIPI4 protein is involved in a process called autophagy that helps remove unnecessary material from cells, including excess iron storage capacity of ferritin Figure 2. Often, the WDR45 gene mutations that cause BPAN syndrome produce the WIPI4 protein lacking the desired function. As a result, the autophagy process is impaired, and purification of unnecessary materials from the cells is also impaired. Researchers believe that nerve cells (neurons) may be vulnerable to autophagy dysfunction, in which dendrites and axons are damaged, which leads to difficulty in transporting and digesting waste and recycling them [1-7]. By lysozems. As a result, waste can be created and damaged in 
these areas. Damage to neurons causes neural problems in BPAN syndrome. BPAN syndrome follows the dominant X-inheritance pattern. The gene associated with this situation is located on chromosome $\mathrm{X}$, which is one of two sex chromosomes. In women (which have two chromosomes $\mathrm{X}$ ), mutation in one of two versions of the gene in each cell is sufficient to disrupt it. In men (which have only one chromosome X), mutations in the single copy of the gene in each cell are disrupted. Hereditary attributes associated with X are that fathers cannot pass attributes associated with $\mathrm{X}$ to their son's sons. In most dominant X-linked disorders, men have more severe symptoms than women Figure 3. While this is not always the case with BPAN syndrome, since most people with this disorder are women, this is most likely due to the fact that a few men affected by BPAN syndrome live up to the age of puberty and marriage. Almost all cases of BPAN syndrome are due to new mutations in the WDR45 gene and occur in people with no history of family impairment. In rare cases, a person with BPAN syndrome receives a mutated allele from a mildly mild woman. Studies show that men with BPAN syndrome and most women with BPAN syndrome do not have a child.

\section{Frequency of BPAN Syndrome}

BPAN syndrome is a rare genetic disorder whose frequency is not known in the world. So far, about 100 cases of this syndrome have been reported in medical literature from around the world. BPAN syndrome seems to account for about $1-2 \%$ of all NBIA disorders.

\section{Diagnosis of BPAN Syndrome}

BPAN syndrome is diagnosed based on the clinical and physical findings of patients and some pathologic and neurological tests. The most accurate method for detecting this syndrome is the molecular genetic testing of the WDR45 gene to investigate the presence of possible mutations. BPAN syndrome treatment routes. The BPAN syndrome treatment and management strategy is symptomatic and supportive. Treatment may be done by a team of experts, including a neurologist, orthopedic specialist, pharyngologist, pulmonary specialist, and other healthcare professionals. There is no standard treatment for this syndrome and all clinical measures are needed to reduce the suffering of the infected person. Genetic counseling is also a special place for all parents who want a healthy baby.

\section{References}

1. Asadi Shahin, Shiva Gholizadeh, Mahsa Jamali, Shahriar Alipour, Leyla Javadi, et al (2018) Pathology in Medical Genetics Book 11 Amidi Publications (A-H) chapter 2 (B): 153-160.

2. Ebrahimi Fakhari D, Saffari A, Wahlster L, Lu J, Byrne S, Hoffmann GF, et al. (2016) Congenital disorders of autophagy: an emerging novel class of inborn errors of neuro-metabolism Brain 139(2): 317-337.

3. Gregory A, Kurian MA, Haack T, Hayflick SJ, Hogarth P (2017) BetaPropeller Protein-Associated Neurodegeneration. In: Pagon RA, Adam MP, Ardinger HH, Wallace SE, Amemiya A, et al. (Eds.). Gene Reviews ${ }^{\circledR}$, University of Washington, Seattle, USA.

4. Haack TB, Hogarth P, Gregory A, Prokisch H, Hayflick SJ (2013) BPAN: the only X-linked dominant NBIA disorder. Int Rev Neurobiol 110: 85-90.

5. Hayflick SJ, Kruer MC, Gregory A, Haack TB, Kurian MA et al. (2013) $\beta$-Propeller protein-associated neurodegeneration: a new X-linked dominant disorder with brain iron accumulation. Brain 136(Pt 6): 17081717.

6. Nishioka K, Oyama G, Yoshino H, Li Y, Matsushima T, et al. (2015) High frequency of beta-propeller protein-associated neurodegeneration (BPAN) among patients with intellectual disability and young-onset parkinsonism. Neurobiol Aging 36(5): e9-2004-e2015.

7. Saitsu H, Nishimura T, Muramatsu K, Kodera H, Kumada S, et al. (2013) De novo mutations in the autophagy gene WDR45 cause static encephalopathy of childhood with neurodegeneration in adulthood. Nat Genet 45(4): 445-449.

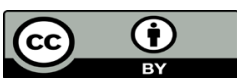

This work is licensed under Creative Commons Attribution 4.0 License

To Submit Your Article Click Here: Submit Article

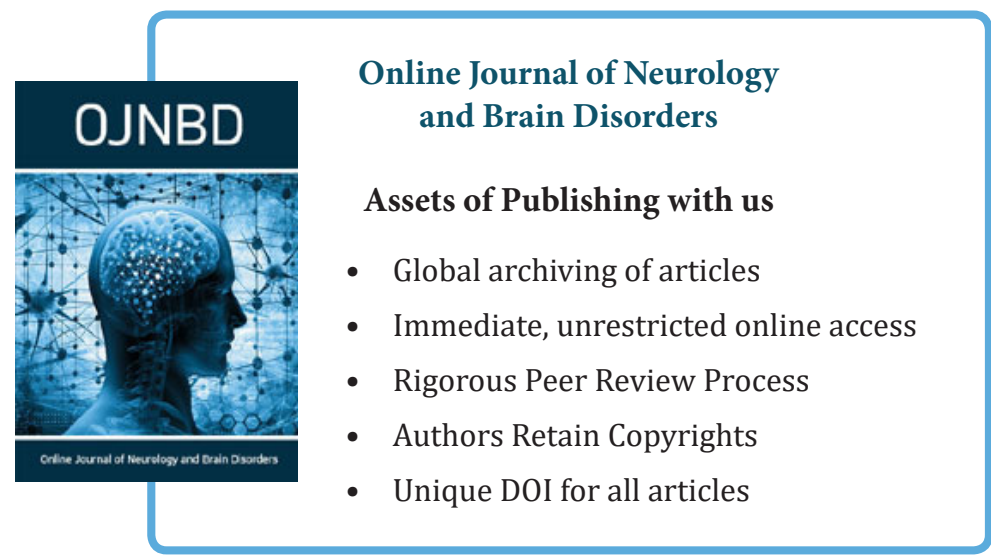

